\title{
ENUMERATION OF SURFACES CONTAINING AN ELLIPTIC QUARTIC CURVE
}

\author{
F. CUKIERMAN, A. F. LOPEZ, AND I. VAINSENCHER \\ (Communicated by Lev Borisov)
}

Dedicated to Steve Kleiman on the occasion of his 70th birthday

\begin{abstract}
A very general surface of degree at least four in $\mathbb{P}^{3}$ contains no curves other than intersections with surfaces. We find a formula for the degree of the locus of surfaces in $\mathbb{P}^{3}$ of degree at least five which contain some elliptic quartic curves. We also compute the degree of the locus of quartic surfaces containing an elliptic quartic curve, a case not covered by that formula.
\end{abstract}

\section{INTRODUCTION}

The Noether-Lefschetz theorem asserts that all curves contained in a very general surface $F$ of degree at least four in $\mathbb{P}^{3}$ are complete intersections. This is usually rephrased by saying that the Picard group is $\mathbb{Z}$. Noether-Lefschetz theory shows that, roughly speaking, each additional generator for Pic $F$ decreases the dimension of the locus of such $F$ in $\mathbb{P}^{N}=\left|\mathcal{O}_{\mathbb{P}^{3}}(d)\right|, d \geq 4$.

Let $W$ be a closed, irreducible subvariety of the Hilbert scheme of curves in $\mathbb{P}^{3}$ with Hilbert polynomial $p_{W}(t)$. Let us denote by $N L(W, d)$ the subset of $\mathbb{P}^{N}$ defined by the requirement that the surface contain some member of $W$.

The purpose of this note is to address the question of determining the degree of $N L(W, d)$ for the family of elliptic quartic curves in $\mathbb{P}^{3}$.

When $W$ is the family of lines, or conics, or twisted cubics, formulas for $N L(W, d)$ have been found in [9]. There as here, we follow the strategy of using Bott's formula as explained in 5. We get a polynomial formula (4.3) valid for $d \geq 5$. We also compute the degree $(\mathbf{3 8 4 7 5})$ of the locus of quartic surfaces containing an elliptic quartic curve. The case of quartic surfaces is not covered by the formula essentially because the map that forgets the curve shrinks dimensions: generically, it contracts a pair of disjoint pencils; see 4.2 .

Received by the editors November 15, 2011 and, in revised form, August 20, 2012 and September 19, 2012.

2010 Mathematics Subject Classification. Primary 14N05, 14N15; Secondary 14C05.

Key words and phrases. Intersection theory, Noether-Lefschetz locus, enumerative geometry.

The first author was partially supported by CONICET-Argentina.

The second author was partially supported by PRIN Geometria delle varietà algebriche e dei loro spazi di moduli.

The third author was partially supported by CNPQ-Brasil. 


\section{There is A POLYNOMial FORMUlA}

Let $W$ be a closed, irreducible subvariety of the Hilbert scheme of curves in $\mathbb{P}^{3}$ with Hilbert polynomial $p_{W}(t)$. Let

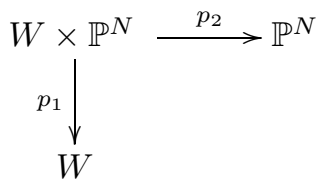

denote the projection maps from $W \times \mathbb{P}^{N}$. Castelnuovo-Mumford regularity 12 shows that for all $d \gg 0$, the subset $\widetilde{N L}(W, d)$ of pairs $(C, F)$ in $W \times \mathbb{P}^{N}$ such that the curve $C$ is contained in the surface $F$ is a projective bundle over $W$ via $p_{1}$. We have

$$
\operatorname{codim}_{W \times \mathbb{P}^{N}} \widetilde{N L}(W, d)=p_{W}(d) .
$$

For instance, if $W$ is the Grassmannian of lines in $\mathbb{P}^{3}$, then $p_{W}(d)=d+1$ and so $\operatorname{dim} \widetilde{N L}(W, d)=N-(d-3), d \geq 1$.

Let us denote by $N L(W, d)$ the subset of $\mathbb{P}^{N}$ defined by the requirement that the surface contain some member of $W$. In other words, with notation as in (2.1),

$$
N L(W, d)=p_{2}(\widetilde{N L}(W, d)) .
$$

We assume henceforth that the general member of $W$ is a smooth curve.

Proposition 2.1. For fixed $W$ we have that $\operatorname{deg} N L(W, d)$ is a polynomial in $d$ of degree $\leq 3 \operatorname{dim} W$, for all $d \gg 0$.

Proof. Let $\widetilde{C} \subset W \times \mathbb{P}^{3}$ be the universal curve. Likewise, let $\widetilde{F} \subset \mathbb{P}^{N} \times \mathbb{P}^{3}$ be the universal surface of degree $d$. Write $\widehat{C}, \widehat{F}$ for their pullbacks to $W \times \mathbb{P}^{N} \times \mathbb{P}^{3}$. We have the diagram of sheaves over $Y:=W \times \mathbb{P}^{N} \times \mathbb{P}^{3}$ :

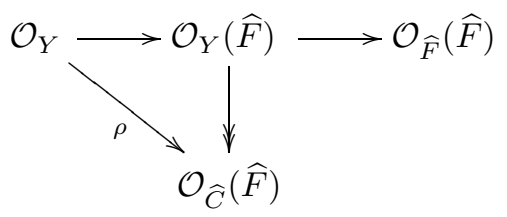

By construction, the slant arrow $\rho$ vanishes at a point $(C, F, x) \in W \times \mathbb{P}^{N} \times \mathbb{P}^{3}$ if and only if $x \in F \cap C$. We have $C \subset F$ when the previous condition holds for all $x \in C$ (point with values in any $\mathbb{C}$-algebra). Thus $\widehat{N L}(W, d)$ is equal to the scheme of zeros of $\rho$ along the fibers of the projection $p_{12}: \widehat{C} \rightarrow W \times \mathbb{P}^{N}$. Recalling [1. (2.1), p.14], this is the same as the zeros of the adjoint section of the direct image vector bundle $p_{12 \star}\left(\mathcal{O}_{\widehat{C}}(\widehat{F})\right)$. Let

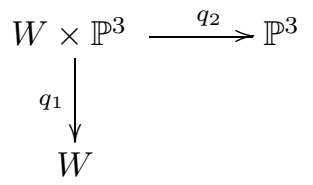

denote the projection maps from $W \times \mathbb{P}^{3}$. Since $\mathcal{O}(\widetilde{F})=\mathcal{O}_{\mathbb{P}^{N}}(1) \otimes \mathcal{O}_{\mathbb{P}^{3}}(d)$, by the projection formula we have to make do with a section of $\mathcal{O}_{\mathbb{P}^{N}}(1) \otimes \mathcal{E}_{d}$, where

$$
\mathcal{E}_{d}=q_{1 \star}\left(\mathcal{O}_{\widetilde{C}}(d)\right) \text {. }
$$


By Castelnuovo-Mumford and base change theory, there is an integer $d_{0}$ such that $\mathcal{E}_{d}$ is a vector bundle of rank $p_{W}(d)$ for all $d \geq d_{0}$ (=regularity; see Remarks 2.2.). In fact, it fits into the exact sequence of vector bundles over $W$,

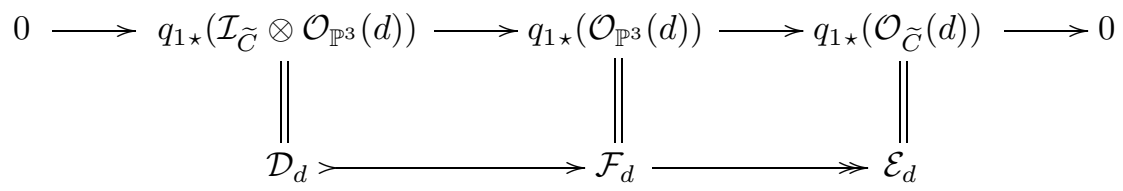

where we set for short

$$
\mathcal{F}_{d}=H^{0}\left(\mathcal{O}_{\mathbb{P}^{3}}(d)\right)
$$

(trivial vector bundle with fiber) the space of polynomials of degree $d$. Taking the projectivization and pulling back to $\mathbb{P}^{N} \times W$, we get

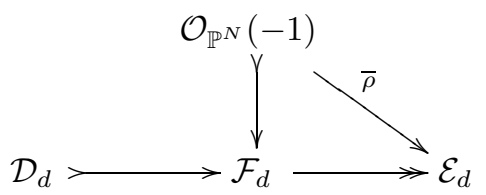

By construction, $\bar{\rho}$ vanishes precisely over $\widetilde{N L}(W, d)$. This shows that we actually get

$$
\widetilde{N L}(W, d)=\mathbb{P}\left(\mathcal{D}_{d}\right) .
$$

Since the rank of $\mathcal{E}_{d}$ and codimension of $\widetilde{N L}(W, d)$ agree, it follows that $\widetilde{N L}(W, d)$ represents the top Chern class of $\mathcal{O}_{\mathbb{P}^{N}}(1) \otimes \mathcal{E}_{d}$ (cf. [6, 3.2.16, p. 61]). This is the key to the calculation of degrees below. The map

$$
\begin{array}{rll}
W \times \mathbb{P}^{N} \supset \widetilde{N L}(W, d) & \stackrel{p_{2}}{\longrightarrow} & N L(W, d) \subset \mathbb{P}^{N} \\
(C, F) & \mapsto & F
\end{array}
$$

is generically injective by Noether-Lefschetz theory [8] cf. Corollary 4.2 below. Therefore the degree of $N L(W, d)$ can be computed upstairs. Namely, setting

$$
m=\operatorname{dim} \widetilde{N L}(W, d), H=c_{1} \mathcal{O}_{\mathbb{P}^{N}}(1),
$$

we have

$$
\operatorname{deg} N L(W, d)=\int H^{m} \cap \widetilde{N L}(W, d)=\int H^{m} c_{\tau}\left(\mathcal{O}_{\mathbb{P}^{N}}(1) \otimes \mathcal{E}_{d}\right),
$$

where $\tau=\operatorname{rank} \mathcal{E}_{d}$. Expanding the top Chern class and pushing forward to $W$, we arrive at

$$
\operatorname{deg} N L(W, d)=\int_{W} c_{w}\left(\mathcal{E}_{d}\right)
$$

with $w=\operatorname{dim} W$. Since $\mathcal{E}_{d}$ is the pushforward of a sheaf on $W \times \mathbb{P}^{3}$, we may apply Grothendieck-Riemann-Roch [6, p. 286] to express the Chern character of $\mathcal{E}_{d}$ as

$$
\operatorname{ch}\left(\mathcal{E}_{d}\right)=\operatorname{ch}\left(q_{1 !}\left(\mathcal{O}_{\widetilde{C}}(d)\right)=q_{1 \star}\left(\operatorname{ch}\left(\mathcal{O}_{\widetilde{C}}\right) \operatorname{ch}\left(\mathcal{O}_{\mathbb{P}^{3}}(d)\right) \operatorname{todd} \mathbb{P}^{3}\right) .\right.
$$

Notice that the right hand side is a polynomial in $d$ of degree $\leq 3$. Since $c_{w}$ is a polynomial of degree $w$ on the coefficients of the Chern character, we deduce that $c_{w}\left(\mathcal{E}_{d}\right)$ is a polynomial in $d$ of degree $\leq 3 w$. 
Remarks 2.2. (1) The assertion that $\mathcal{E}_{d}$, as defined in (2.2), is a vector bundle of rank $p_{W}(d)$ holds for all $d$ beyond $d_{0}=$ the maximal Castelnuovo-Mumford regularity of the members of $W$. For instance, if $W$ is the family of lines in $\mathbb{P}^{3}$, then $d_{0}=1$.

(2) For the case of elliptic quartic curves presented below, we note that the regularity of the ideals $\left\langle x_{1}^{2}, x_{2}^{2}\right\rangle$ and $\left\langle x_{1} x_{2}, x_{1}^{2}, x_{2}^{3}\right\rangle$ is 3 , whereas for $\left\langle x_{0}^{2}, x_{0} x_{1}, x_{0} x_{2}^{2}, x_{1}^{4}\right\rangle$ it is 4 . The last two ideals are representatives of the closed orbits in $W$. An argument of semi-continuity shows that $d_{0}=4$ works for all members of $W$; see [2]. Nevertheless, the map in (2.5) is generically injective only for $d \geq 5$; cf. (4.2) below. Notice that the full Hilb ${ }^{4 t} \mathbb{P}^{3}$ has a "ghost" component with regularity index $\geq 5$; see [7].

\section{ElLIPTIC QUARTICS}

We consider now the case of surfaces of degree $\geq 4$ containing an elliptic quartic curve in $\mathbb{P}^{3}$. Thus, a general member $C_{4}$ of $W$ is the intersection of two quadric surfaces. The parameter space $W$ is described in [2] and has been used in [5] to enumerate curves in Calabi-Yau 3-folds. For the convenience of the reader, we summarize below its main features.

The Noether-Lefschetz locus of quartic surfaces containing some $C_{4}$ is slightly exceptional. This is a case when the map (2.5) fails to be generically injective (cf. Corollary 4.2): it actually shrinks $\operatorname{dim} \widetilde{N L}(W, d)=34$ to $\operatorname{dim} N L(W, d)=33$. Indeed, if a quartic surface $F$ contains some general elliptic quartic $C_{4}$, then $F$ must contain the two pencils $\left|C_{4}\right|$ and $\left|C_{4}^{\prime}\right|$, where $C_{4}^{\prime}$ is the residual intersection of $F$ with a quadric containing $C_{4}$, i.e., $2 H=C_{4}+C_{4}^{\prime}$, with $H=$ plane section. We show in 3.2 that $N L(W, 4)$ is a hypersurface of degree 38475 in $\mathbb{P}^{34}=\left|\mathcal{O}_{\mathbb{P}^{3}}(4)\right|$.

3.1. Next we give an outline of the calculation. Put

$$
\mathbb{X}=\mathbb{G}\left(2, \mathcal{F}_{2}\right),
$$

the Grassmannian of pencils of quadrics in $\mathbb{P}^{3}$.

The diagram below summarizes the construction of $W$ as explained in [2]:

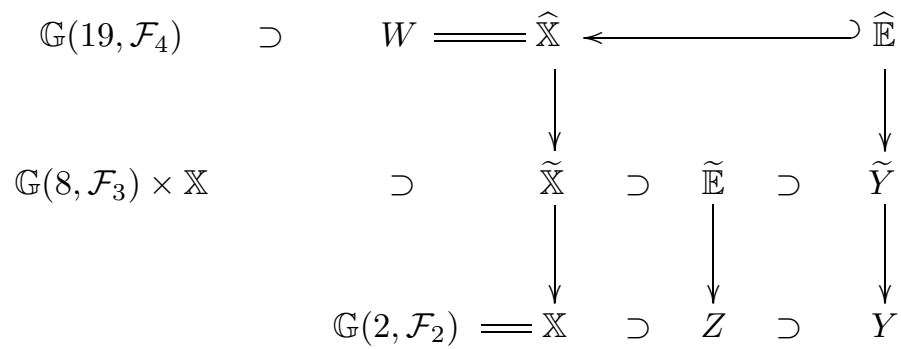

where

$$
\left\{\begin{array}{l}
Z \cong \check{\mathbb{P}}^{3} \times \mathbb{G}\left(2, \mathcal{F}_{1}\right) \text { consists of pencils with a fixed plane; } \\
Y \cong\{(p, \ell) \mid p \supset \ell\}=\text { closed orbit of } Z ; \\
\widetilde{Y} \longrightarrow Y=\mathbb{P}^{2}-\text { bundle of degree } 2 \text { divisors on the varying } \ell \subset p \\
\widehat{X}=\text { the blow-up of } \widetilde{X} \text { along } \widetilde{Y} ; \text { and } \\
\widetilde{X}=\text { the blow-up of } X \text { along } Z
\end{array}\right.
$$




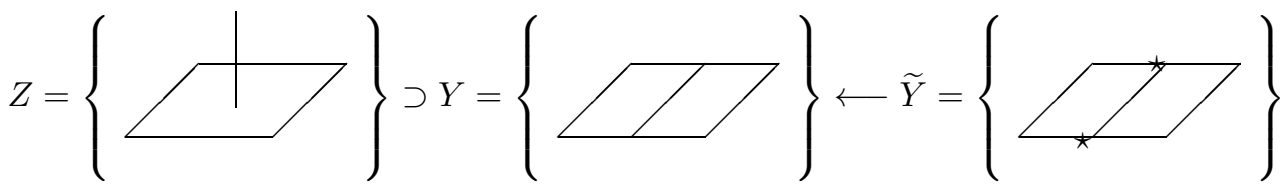

Let

$$
\mathcal{A} \subset \mathcal{F}_{2} \times \mathbb{X}
$$

be the tautological subbundle of rank 2 over our Grassmannian of pencils of quadrics. There is a natural map of vector bundles over $\mathbb{X}$ induced by multiplication,

$$
\mu_{3}: \mathcal{A} \otimes \mathcal{F}_{1} \longrightarrow \mathcal{F}_{3} \times \mathbb{X}
$$

with generic rank 8 . It drops rank precisely over $Z$. It induces a rational map $\kappa: \mathbb{X} \rightarrow \mathbb{G}\left(8, \mathcal{F}_{3}\right)$. Blowing up $\mathbb{X}$ along $Z$, we find the closure $\widetilde{\mathbb{X}} \subset \mathbb{G}\left(8, \mathcal{F}_{3}\right) \times \mathbb{X}$ of the graph of $\kappa$. Similarly, up on $\widetilde{\mathbb{X}}$ we have a subbundle $\mathcal{B} \subset \mathcal{F}_{3} \times \widetilde{\mathbb{X}}$ of rank 8 and a multiplication map

$$
\mu_{4}: \mathcal{B} \otimes \mathcal{F}_{1} \longrightarrow \mathcal{F}_{4} \times \widetilde{\mathbb{X}}
$$

with generic rank 19. The scheme of zeros of $\bigwedge^{19} \mu_{4}$ is equal to $\tilde{Y}$. Indeed, it can be checked that each fiber of $\mathcal{B}$ is a linear system of cubics which

- either has base locus equal to a curve with Hilbert polynomial $p_{W}(t)=4 t$

- or is of the form $p \cdot \mathcal{F}_{2}^{\star \star}$, meaning the linear system with fixed component a plane $p$, and $\mathcal{F}_{2}^{\star \star}$ denoting an 8-dimensional space of quadrics which define a subscheme of $p$ of dimension 0 and degree 2 .

The exceptional divisor $\widehat{\mathbb{E}}$ is a $\mathbb{P}^{8}$-bundle over $\widetilde{Y}$. The fiber of $\widehat{\mathbb{E}}$ over $\left(p, y_{1}+\right.$ $\left.y_{2}\right) \in \tilde{Y}$ is the system of quartic curves in the plane $p$ which are singular at the doublet $y_{1}+y_{2}$. Precisely, if $x_{0}, \ldots, x_{3}$ denote homogeneous coordinates on $\mathbb{P}^{3}$, assuming $p:=x_{0}, \ell=\left\langle x_{0}, x_{1}\right\rangle$, a typical doublet has homogeneous ideal of the form $\left\langle x_{0}, x_{1}, f\left(x_{2}, x_{3}\right)\right\rangle$, with $\operatorname{deg} f=2$. Our system of plane quartics lies in the ideal $\left\langle x_{1}, f\right\rangle^{2}=\left\langle x_{1}^{2}, x_{1} f, f^{2}\right\rangle$. Given a non-zero quartic $g$ in this ideal, we may form the ideal $J=\left\langle x_{0}^{2}, x_{0} x_{1}, x_{0} f, g\right\rangle$. It can be checked that $J$ contains precisely 19 independent quartics and its Hilbert polynomial is correct. In fact, any such ideal is 4-regular (in the sense of Castelnuovo-Mumford). Moreover, upon $\widehat{\mathbb{X}}$ we get a subbundle

$$
\mathcal{C} \subset \mathcal{F}_{4} \times \widehat{\mathbb{X}}
$$

of rank 19. Each of its fibers over $\widehat{\mathbb{X}}$ is a system of quartics that cut out a curve with the correct Hilbert polynomial. The multiplication map

$$
\mathcal{C} \otimes \mathcal{F}_{d-4} \longrightarrow \mathcal{F}_{d} \times \widehat{\mathbb{X}}
$$

is of constant rank $\left(\begin{array}{c}d+3 \\ 3\end{array}\right)-4 d$. The image

$$
\mathcal{D}_{d} \subset \mathcal{F}_{d} \times \widehat{\mathbb{X}}
$$

is a subbundle as in (2.3). We have

$$
\widetilde{N L}(W, d)=\mathbb{P}\left(\mathcal{D}_{d}\right) \subset \mathbb{P}^{N} \times \widehat{\mathbb{X}} .
$$

Now the map $\widetilde{N L}(W, d) \rightarrow N L(W, d)$ is generically injective for $d \geq 5$ in view of Corollary 4.2 (ii) below. Hence the degree of the image $N L(W, d) \subset \overline{\mathbb{P}}^{N}$ is given by $\int c_{16} \mathcal{E}_{d}$; cf. (2.6). 
The above description suffices to feed in Bott's localization formula with all the required data. Indeed, $\widehat{\mathbb{X}}$ inherits a $\mathbb{C}^{\star}$-action, with (a lot) of isolated fixed points. The vector bundle $\mathcal{D}_{d} \rightarrow \widehat{\mathbb{X}}$ is equivariant; ditto for $\mathcal{E}_{d}$. Bott's formula reads [4]

$$
\int c_{16} \mathcal{E}_{d}=\sum_{p \in \text { fixpts }} \frac{c_{16}^{T}\left(\mathcal{E}_{d}\right)_{p}}{c_{16}^{T} \tau_{p}}
$$

The equivariant classes on the r.h.s are calculated in two steps. Below we set $d=5$ for simplicity. First, find the $\mathbb{C}^{\star}$-weight decomposition of the fibers of the vector bundles $\mathcal{E}_{d}$ and $\tau$ at each fixed point. Then, since the fixed points are isolated, the equivariant Chern classes $c_{i}^{T}$ are just the symmetric functions of the weights.

For instance, for the tangent bundle $\tau$, say at the fixed point corresponding to the pencil $p=\left\langle x_{0}^{2}, x_{1}^{2}\right\rangle \in \mathbb{X}$, we have

$$
\tau_{p}=\operatorname{Hom}\left(p, \mathcal{F}_{2} / p\right)=p^{\vee} \otimes \mathcal{F}_{2} / p=\frac{x_{0} x_{1}}{x_{0}^{2}}+\frac{x_{0} x_{2}}{x_{0}^{2}}+\frac{x_{1} x_{2}}{x_{0}^{2}}+\cdots+\frac{x_{3}^{2}}{x_{1}^{2}} .
$$

Each of the 16 fractions, $\frac{x_{i} x_{j}}{x_{k} x_{l}}$, on the right hand side symbolizes a 1-dimensional subspace of $\tau_{p}$ where $\mathbb{C}^{\star}$ acts with character $t^{x_{i}+x_{j}-x_{k}-x_{l}}$. The denominator in (3.2) is the corresponding equivariant top Chern class, to wit

$$
\left(x_{1}-x_{0}\right)\left(x_{2}-x_{0}\right)\left(x_{1}+x_{2}-2 x_{0}\right) \cdots\left(2 x_{3}-2 x_{1}\right) .
$$

The numerator in (3.2) requires finding the monomials of degree 5 that survive modulo the ideal

$$
\left\langle x_{0}^{2}, x_{1}^{2}\right\rangle \cdot\left\langle x_{0}, \ldots, x_{3}\right\rangle^{3} .
$$

We are left with $20\left(=\operatorname{rank} \mathcal{E}_{5}\right)$ terms:

$$
x_{2}^{3} x_{1} x_{0}+x_{3} x_{2}^{3} x_{1}+\cdots+x_{3}^{4} x_{0} .
$$

Now the equivariant Chern class $c_{16}^{T}\left(\mathcal{E}_{d}\right)_{p}$ is the coefficient of $t^{16}$ in the product

$$
\left(t+x_{0}+x_{1}+3 x_{2}\right)\left(t+x_{1}+3 x_{2}+x_{3}\right) \cdots\left(t+x_{0}+4 x_{3}\right)
$$

(20 factors). In practice, all these calculations are made by substituting $x_{i}$ for suitable numerical values; cf. the computer algebra scripts in [13].

3.2. The case $d=4$. Presently $p_{2}: \widetilde{N L}(W, 4) \rightarrow N L(W, 4)$ is no longer generically injective. It shrinks dimension by one: a general fiber is a disjoint union of $\mathbb{P}^{1}$ 's (cf. Corollary 4.2(iii) below). Explicitly, say $F_{4}=A_{1} Q_{1}+A_{2} Q_{2}, \operatorname{deg} A_{i}=$ $\operatorname{deg} Q_{i}=2$, with $A_{i}$ and $Q_{i}$ generic. Then

$$
F_{4}=\left(A_{1}-t Q_{2}\right) Q_{1}+\left(A_{2}+t Q_{1}\right) Q_{2},
$$

so $F_{4}$ contains the pencil of elliptic quartics $\left\langle A_{1}-t Q_{2}, A_{2}+t Q_{1}\right\rangle, t \in \mathbb{P}^{1}$; setting $t=\infty$, we find $\left\langle Q_{1}, Q_{2}\right\rangle$. Similarly, we get $\left\langle Q_{1}-t A_{2}, Q_{2}+t A_{1}\right\rangle$. This is one and the same pencil. But there is also $\left\langle A_{1}-t A_{2}, Q_{2}+t Q_{1}\right\rangle$. In general, these 2 pencils are disjoint. Looking at them as curves in $\mathbb{X}=\mathbb{G}\left(2, \mathcal{F}_{2}\right)$, we actually get a Plückerembedded conic, $\left(A_{1}-t A_{2}\right) \wedge\left(Q_{2}+t Q_{1}\right)=A_{1} \wedge Q_{2}+t\left(A_{1} \wedge Q_{1}-A_{2} \wedge Q_{2}\right)-t^{2} A_{2} \wedge Q_{1}$, disjoint from $Y$ (see (3.1)). In particular, capping each conic against the Plücker hyperplane class $\Pi=-c_{1} \mathcal{A}$, we find 2 . As before, we may write

$$
\operatorname{deg} N L(W, d)=\int H^{33} \cap N L(W, d) .
$$


The cycle $p_{2}^{\star} H^{33} \cap N L(W, d)$ can be represented by a sum of $\operatorname{deg} N L(W, d)$ disjoint unions of pairs of $\mathbb{P}^{1}$ 's. Hence

$$
\begin{array}{r}
\operatorname{deg} N L(W, d)=\frac{1}{4} \int_{\widehat{\mathbb{X}}} \Pi \cdot H^{33} \cap \widetilde{N L}(W, d)=\frac{1}{4} \int_{\widehat{\mathbb{X}}} \Pi \cdot\left(p_{1}\right)_{\star} H^{33} \cap \widetilde{N L}(W, d) \\
=\frac{1}{4} \int_{\widehat{\mathbb{X}}} \Pi \cdot c_{15}\left(\mathcal{E}_{4}\right) .
\end{array}
$$

The latter integral can be computed via Bott's formula, and we get 38475; cf. the script in [13. This has been found independently in [3] with different techniques, using [10].

\section{THE FIBERS OF $p_{2}$}

The main result needed to validate the above enumeration is the following.

Proposition 4.1. Let $C \subset \mathbb{P}^{3}$ be a smooth irreducible curve of degree e and genus g. Let $d \gg 0$ and let $F \subset \mathbb{P}^{3}$ be a general surface of degree $d$ containing $C$. Then $C$ is the only effective divisor of degree $e$ and arithmetic genus $g$ on $F$.

Proof. By [8, Cor. II.3.8] we have that $\operatorname{Pic}(F)$ is freely generated by its hyperplane section $H$ and $C$. Let $C^{\prime}$ be an effective divisor of degree $e$ and arithmetic genus $g$ on $F$. Then there are two integers $a, b$ such that, on $F$, we have $C^{\prime} \sim a H+b C$. Now $e=H \cdot C^{\prime}=a d+b e$, so that

$$
a=\frac{e}{d}(1-b)
$$

By the adjunction formula we have

$$
C^{2}=2 p_{a}(C)-2-K_{F} \cdot C=2 g-2-e(d-4)=\left(C^{\prime}\right)^{2}<0
$$

as $d \gg 0$. Now

$$
C^{2}=\left(C^{\prime}\right)^{2}=(a H+b C)^{2}=a^{2} d+b^{2} C^{2}+2 a b e,
$$

and using (4.1) we get

$$
\left(1-b^{2}\right)\left(e^{2}-d C^{2}\right)=0 .
$$

Note that $e^{2}-d C^{2}>0$ by (4.2), whence $b= \pm 1$. If $b=-1$ we have from (4.1) $a=\frac{2 e}{d} \notin \mathbb{Z}$, as $d \gg 0$. Therefore we deduce that $b=1$ and $a=0$, that is, $C^{\prime} \sim C$. Since $C^{2}<0$ we must have $C^{\prime}=C$.

Corollary 4.2. Let $W$ be an irreducible subvariety of a Hilbert scheme component of curves in $\mathbb{P}^{3}$ of degree $e$ and arithmetic genus $g$ with general member smooth. Let $\mathbb{P}^{N}=\mathbb{P}\left(H^{0}\left(\mathcal{O}_{\mathbb{P}^{3}}(d)\right)\right)$. Then:

(i) There is a $d_{0}$ such that for all $d \geq d_{0}$ the projection map $p_{2}: \widetilde{N L}(W, d) \rightarrow \mathbb{P}^{N}$ is generically injective.

(ii) If $W$ is the family of elliptic quartics, then we can take $d_{0}=5$; i.e., $p_{2}$ is generically one-to-one for $d \geq 5$.

(iii) For $d=4$, the general fiber of $\widetilde{N L}(W, 4) \stackrel{p_{2}}{\longrightarrow} N L(W, 4)$ is two disjoint $\mathbb{P}^{1}$ 's.

Proof. We know from (2.4) that $p_{1}: \widetilde{N L}(W, d) \rightarrow W$ is a projective bundle. Hence $N L(W, d)=p_{2}(\widetilde{N L}(W, d))$ is irreducible, and a general element $F \in N L(W, d)$ can be identified with a general hypersurface of degree $d$ containing a general $C \in W$. Hence assertion (i) follows from the proposition. Assertion (ii) also follows, except for $d=8$. In this case, with the notation as in (4.1), if $b=-1$ we would get $a=1$, 
whence $C^{\prime} \sim H-C$, so that $C$ would be contained in a plane, which is absurd. If $b=1$, we may proceed as at the end of the proof of the above proposition.

If $d=4$, then we get instead $C^{\prime} \sim 2 H-C$. The exact sequence

$$
0 \longrightarrow \mathcal{O}_{F} \longrightarrow \mathcal{O}_{F}(C) \longrightarrow \mathcal{O}_{C}(C)=\mathcal{O}_{C} \longrightarrow 0
$$

shows that $\left|\mathcal{O}_{F}(C)\right| \cong \mathbb{P}^{1}$ and similarly $\left|\mathcal{O}_{F}(2 H-C)\right| \cong\left|\mathcal{O}_{F}\left(C^{\prime}\right)\right| \cong \mathbb{P}^{1}$. Moreover, there is no curve $D$ on $F$ such that $D \sim C$ and $D \sim 2 H-C$, for then $2 C \sim 2 H$, giving the contradiction $0=C^{2}=H^{2}=4$. This proves that, in this case, the general fiber of $p_{2}$ is two disjoint $\mathbb{P}^{1}$ 's:

$$
p_{2}^{-1}(F)=\left|\mathcal{O}_{F}(C)\right| \cup\left|\mathcal{O}_{F}(2 H-C)\right| .
$$

4.1. The formula. In view of Proposition 2.1 it suffices to find the degrees of $N L(W, d)$ for $3 \cdot 16+1$ values of $d \geq 5$ and interpolate. This is done in [13. We obtain

$$
\begin{gathered}
\left(\begin{array}{c}
d-2 \\
3
\end{array}\right)\left(106984881 d^{29}-3409514775 d^{28}+57226549167 d^{27}\right. \\
-643910429259 d^{26}+5267988084411 d^{25}-31628193518727 d^{24}+126939490699539 d^{23} \\
-144650681793207 d^{22}-2701978741671631 d^{21}+28913126128882647 d^{20} \\
-182919422241175163 d^{19}+858473373993063183 d^{18}-3061191057059772423 d^{17} \\
+7448109470245631187 d^{16}-3841505361473930575 d^{15}-80644842327962348733 d^{14} \\
+568059231910087276234 d^{13}-2560865812030993315212 d^{12}+9159430737614259196104 d^{11} \\
-27608527286339077691280 d^{10}+71605637662357479581024 d^{9} \\
-160009170853633152594240 d^{8}+303685692157317249665152 d^{7} \\
-473993548940769326728704 d^{6}+571505502502703378479104 d^{5} \\
-459462480152611231457280 d^{4}+111908571251948243582976 d^{3} \\
+251116612534424272896000 d^{2}-328452832055501940326400 d \\
+136886449647246114816000) /\left(2^{27} \cdot 3^{9} \cdot 5^{2} \cdot 7^{2} \cdot 11 \cdot 13\right) .
\end{gathered}
$$

\section{ACKNOWLEDGMEnTS}

The authors thank the referee for kindly pointing out to them a sizable portion of the argument that required clarification. They are also grateful to Kristian Ranestad and Rahul Pandharipande for correcting an error in $₫ 3.2$ and for calling their attention to [3] and [10].

\section{REFERENCES}

[1] Allen B. Altman and Steven L. Kleiman, Foundations of the theory of Fano schemes, Compositio Math. 34 (1977), no. 1, 3-47. MR0569043 (58 \#27967)

[2] Israel Vainsencher and Dan Avritzer, Compactifying the space of elliptic quartic curves, Complex projective geometry (Trieste, 1989/Bergen, 1989), London Math. Soc. Lecture Note Ser., vol. 179, Cambridge Univ. Press, Cambridge, 1992, pp. 47-58. MR1201374 (94c:14027)

[3] Grigoriy Blekherman, Jonathan Hauenstein, John Christian Ottem, Kristian Ranestad, and Bernd Sturmfels, Algebraic boundaries of Hilbert's SOS cones, Compos. Math. 148 (2012), no. 6, 1717-1735. MR2999301

[4] Dan Edidin and William Graham, Localization in equivariant intersection theory and the Bott residue formula, Amer. J. Math. 120 (1998), no. 3, 619-636. MR.1623412 (99g:14005)

[5] Geir Ellingsrud and Stein Arild Strømme, Bott's formula and enumerative geometry, J. Amer. Math. Soc. 9 (1996), no. 1, 175-193, DOI 10.1090/S0894-0347-96-00189-0. MR 1317230 (96j:14039) 
[6] William Fulton, Intersection theory, 2nd ed., Ergebnisse der Mathematik und ihrer Grenzgebiete. 3. Folge. A Series of Modern Surveys in Mathematics [Results in Mathematics and Related Areas. 3rd Series. A Series of Modern Surveys in Mathematics], vol. 2, SpringerVerlag, Berlin, 1998. MR1644323 (99d:14003)

[7] Gerd Gotzmann, The irreducible components of $\mathrm{Hilb}^{4 n}\left(\mathbb{P}^{3}\right)$, arXiv:0811.3160v1 [math.AG], 2008.

[8] Angelo Felice Lopez, Noether-Lefschetz theory and the Picard group of projective surfaces, Mem. Amer. Math. Soc. 89 (1991), no. 438, x+100. MR.1043786 (91f:14030)

[9] José Alberto Maia, Adriana Rodrigues, Fernando Xavier, and Israel Vainsencher, Enumeration of surfaces containing a curve of low degree, preprint, 2011.

[10] Davesh Maulik and Rahul Pandharipande, Gromov-Witten theory and Noether-Lefschetz theory (English summary). A celebration of algebraic geometry, Clay Math. Proc., 18, Amer. Math. Soc., Providence, RI, 2013, pp. 469-507. MR3114953

[11] Paul Meurer, The number of rational quartics on Calabi-Yau hypersurfaces in weighted projective space $P\left(2,1^{4}\right)$, Math. Scand. 78 (1996), no. 1, 63-83. MR.1400851 (98a:14070)

[12] David Mumford, Lectures on curves on an algebraic surface, with a section by G. M. Bergman. Annals of Mathematics Studies, No. 59, Princeton University Press, Princeton, N.J., 1966. MR0209285 (35 \#187)

[13] Israel Vainsencher, computer algebra scripts, http://www.mat.ufmg.br/ israel/Projetos/ $\operatorname{degNL}$ (or arXiv).

Universidad de Buenos Aires, Ciudad Universitaria, Pabellón 1, (1428) Buenos Aires, Argentina

E-mail address: fcukier@dm.uba.ar

Dipartimento di Matematica e Fisica, Università di Roma Tre, Largo San Leonardo Murialdo 1, 00146 Roma, ItAly

E-mail address: lopez@mat.uniroma3.it

iCeX-Departamento de Matemática-UfmG, Av. Antônio Carlos, 6627 - Caixa Postal 702, CEP 31270-901 Belo Horizonte, MG, Brazil

E-mail address: israel@mat.ufmg.br 